\title{
Liver-related Mortality is Increased in Lean Subjects with Non- alcoholic Fatty Liver Disease Compared to Overweight and Obese Subjects
}

\author{
Alexandra Feldman ${ }^{1}$, Bernhard Wernly ${ }^{2}$, Georg Strebinger ${ }^{1}$, Sebastian K. Eder ${ }^{1,3}$, Stephan Zandanell ${ }^{1}$, David \\ Niederseer ${ }^{4,5}$, Michael Strasser ${ }^{1}$, Heike Haufe $^{6}$, Karl Sotlar $^{6}$, Bernhard Paulweber $^{1}$, Christian Datz $^{4}$, Elmar Aigner $^{1}$
}

1) First Department of

Medicine, Paracelsus Medical

University Salzburg, Austria;

2) Second Department of

Medicine, Paracelsus Medical

University Salzburg, Austria;

3) St. Anna Children's

Hospital, Department of

Pediatrics and Adolescent

Medicine, Medical University

of Vienna, Vienna, Austria;

4) Department of Internal

Medicine, Hospital Oberndorf,

Teaching Hospital of the

Paracelsus Medical University Salzburg, Oberndorf, Austria;

5) Department of Cardiology,

University Heart Center

Zurich, University Hospital

Zurich, Zurich, Switzerland;

6) Institute of Pathology,

Paracelsus Medical University,

Salzburg, Austria

\begin{abstract}
Background \& Aims: Although non-alcoholic fatty liver disease (NAFLD) is linked to obesity, a proportion of lean subjects also have NAFLD with potentially distinct clinical features. We studied the outcome of lean NAFLD subjects.

Methods: 299 consecutive patients ( 215 male, 84 female, $49.5 \pm 13.5$ years) with biopsy-proven NAFLD and a follow-up of 8.4 years ( \pm 4.1 ; range: $0.9-18.0$ ) were stratified by body mass index (BMI) at the time of liver biopsy: lean (BMI $\leq 25.0 \mathrm{~kg} / \mathrm{m}, \mathrm{n}=38$ ), overweight (BMI 25.0-29.9 kg/m², $\mathrm{n}=165$ ), obese (BMI $\geq 30.0 \mathrm{~kg} / \mathrm{m}^{2}, \mathrm{n}=93$ ). A control group of 1,013 subjects (547 male, 52.4 \pm 5.8 ) was used for comparison. The time to the event was recorded. Multivariable Cox regression analyses were performed to assess associations with 10 -year-mortality. Hazard ratios (HR) and adjusted hazard ratios (aHR) with 95\% confidence intervals (CI) were calculated.

Results: Age and gender were similar, while components of the metabolic syndrome were less frequent in lean subjects. The proportion of subjects with significant fibrosis and the number of subjects with cirrhosis was increased in lean subjects while the proportion of non-alcoholic steatohepatitis was not different. Mortality in the NAFLD groups was significantly higher than in the control group. Multivariable analysis adjusting for age, gender, and glucose confirmed lower mortality in overweight (aHR 0.21; 95\% CI 0.07-0.62, p=0.005) and in obese (aHR 0.22; 95\% CI 0.06-0.76, p=0.02) compared to lean subjects. Further adjustment for fibrosis weakened the difference between lean and obese $(\mathrm{p}=0.12)$ while the difference to overweight subjects remained intact $(\mathrm{p}=0.01)$. Conclusion: Lean subjects with NAFLD have a high risk of liver-related death. Our data support that lean NAFLD subjects deserve particular attention with regard to clinical follow-up.
\end{abstract}

Key words: non-alcoholic fatty liver disease - BMI - lean - obesity - NASH - end-stage liver disease cardiovascular mortality - outcome.

Abbreviations: aHR: adjusted hazard ratios; BMI: body mass index; CI: confidence interval; csHR: causespecific hazard ratio; FIB-4: Fibrosis-4 score; HCC: hepatocellular carcinoma; HR: hazard ratio; LSM - liver stiffness measurement; MetS: metabolic syndrome; NAFLD: non-alcoholic fatty liver disease; NASH: nonalcoholic steatohepatitis; SAF: steatosis, activity and fibrosis score; US: abdominal ultrasound.

\section{INTRODUCTION}

Non-alcoholic fatty liver disease (NAFLD) affects approximately $25 \%$ of the adult population worldwide, and its rise has paralleled the growing prevalence of obesity [1]. NAFLD has been reliably established as the hepatic manifestation of the metabolic syndrome (MetS) [2]. However, a subgroup of approximately $5-8 \%$ of lean subjects with body mass index
(BMI) $<25.0 \mathrm{~kg} / \mathrm{m}^{2}$ also have NAFLD which has been referred to as "lean NAFLD" [3]. Lean NAFLD appears to be more common among Asians; however, it has been well-reported among Caucasians likewise [4].

Although subjects with lean NAFLD lack the obvious association with obesity, the metabolic profile is similar to subjects with NAFLD and higher BMI. Glucose intolerance, dyslipidemia and visceral obesity are also present in lean NAFLD, there appears to be a particular genetic predisposition which involves liver-specific risk variants such as patatinlike phospholipase domain containing 3 (PNPLA3) or transmembrane 6 superfamily member 2 (TM6SF2) but also variants linked to adipose tissue dysfunction reminiscent of lipodystrophy [5-7]. 
There are conflicting data regarding liver disease severity and the natural outcome of lean NAFLD. On the population level, histological disease severity and worse clinical outcomes in NAFLD are associated with higher degrees of obesity $[8,9]$, but most of these studies have been conducted in Asian cohorts [10]. The stage of fibrosis on liver biopsy has been repeatedly demonstrated to represent the determining factor for the clinical outcome [11]. Of interest, recent reports suggest that lean Caucasian NAFLD subjects may have a severe histological disease [12] and also a high risk for liver-related outcomes [13]. Further, metabolically unhealthy normal weight subjects are at an increased risk of cardiovascular morbidity and mortality similar to obese subjects compared to a metabolically healthy control with similar BMI [14]. These conflicting data may relate to the fact that the lean group has been understudied, underrepresented or even excluded in outcome studies. As this disease group may hold specific genetic, pathophysiological and clinical characteristics, further studies are indicated to assess the translation of these particularities into relevant clinical endpoints.

We have therefore studied our NAFLD cohort by stratification according to BMI with respect to fatal and nonfatal hepatic, cardiovascular or malignant outcomes.

\section{METHODS}

\section{Study Cohorts}

The NAFLD cohort was analyzed retrospectively and included all consecutive subjects who had undergone liver biopsy at the First Department of Internal Medicine, Paracelsus Medical University Salzburg, Austria and the Department of Internal Medicine, Oberndorf Hospital, Austria 1997 - 2017. Of all 466 patients diagnosed with NAFLD [12] outcome data could be retrieved in 343; while 123 of the initial 466 either had missing clinical follow-up data or were counted as lost to follow-up. Furthermore, 44 subjects who had achieved an endpoint before the liver biopsy were also not included. Thus, 299 subjects (215 male, 84 female) aged between 18 and 80 years were studied. As part of the routine work-up, infectious, autoimmune and hereditary causes of liver disease had been ruled out by laboratory tests. Subjects had undergone diagnostic liver biopsy due to (a) evidence of unexplained fatty liver disease and/or (b) persistently elevated liver enzymes and/ or (c) clinical suspicion of advanced liver disease and/or (d) unexplained hyperferritinemia. The diagnosis of NAFLD in lean subjects was reached according to the clinical judgement of the treating hepatologist and retrospective analysis of the clinical data. Hence, the use of potentially steatogenic medication was not allowed as summarized by Roeb et al. [15] and Cataldi et al. [16], while mild variants of lipodystrophy were not systematically excluded and other manifestations of the insulin resistance syndrome such as polycystic ovary syndrome were not investigated as was the presence of obstructive sleep apnea which may represent an aggravating or causative factors for NAFLD in lean, overweight and obese subjects. All subjects were re-evaluated over a time period of approximately 9 months and the last available time point or the time of the respective endpoint that was determined was used for calculation [17].
The control group was taken from the Salzburg Atherosclerosis Prevention Program in subjects at High Individual Risk (the SAPHIR study). This population-based study has been initiated in 1999 as a prospective study to assess factors contributing to vascular diseases [18]. Participants were recruited by screening programs in large companies in and around the city of Salzburg, Austria.

All study participants provided informed consent and the study was approved by the Ethical Committee of Salzburg University. All clinical investigations were conducted according to the principles expressed in the Declaration of Helsinki.

The entire study included 1,770 healthy unrelated Caucasian subjects (663 females and 1,107 males aged 39-67 years) and of these 1,013 could be used as a control group given that a clinical assessment at a follow-up was available. Clinical and biochemical characteristics are reported and clinical endpoints were grouped in an analogous manner as described for the NAFLD group [19].

\section{Clinical and Laboratory Examinations}

Subjects underwent a standard clinical examination and medical history was obtained. Venous blood was drawn for laboratory diagnostics including serum liver tests, viral hepatitis B and $\mathrm{C}$ markers, auto-antibodies, serum iron parameters, copper, ceruloplasmin, inflammation markers, lipid panel and fasting glucose within a month of the liver biopsy. Subjects were excluded if clinical or biochemical signs of cardiac or renal insufficiency, infectious diseases or systemic autoimmune disorders were found. The etiology of liver disease was diagnosed as non-alcoholic if other etiologies were excluded and alcohol was reliably judged below clinically relevant amounts $(<30 \mathrm{~g} / \mathrm{d}$ in males, $<20 \mathrm{~g} / \mathrm{d}$ in females). The presence of metabolic syndrome (MetS) was determined by ATP III criteria [20]. As waist circumference was not available at baseline, the diagnosis of obesity with a BMI $>30$ was counted as a substitute criterion [21,22].

For non-invasive fibrosis estimation, the Fibrosis-4 (FIB-4) score [23] was calculated from baseline data.

Likewise, at the follow-up visit, a careful clinical examination was performed and a medical history was taken for the evaluation of clinical endpoints as specified. Particularly, biochemical measures, abdominal ultrasound (US) and liver stiffness measurement (LSM) (FibroScan ${ }^{\circ}$ ) were performed for the assessment of the stage of liver disease. Subjects were referred to computed tomography or magnetic resonance imaging if hepatocellular carcinoma (HCC) was not reliably ruled out on US. Further, subjects were referred to gastroscopy for assessment of gastroesophageal varices if advanced liver disease was diagnosed on clinical, biochemical or imaging results at the follow-up visit.

\section{Definitions of Fatal and Non-fatal Endpoints}

In order to evaluate the natural course of NAFLD subjects, endpoints for hepatic events, cardiovascular events and extrahepatic malignancies were defined as fatal and non-fatal for each category. Total events were defined as fatal and nonfatal events combined.

Hepatic fatal endpoints were defined as liver-related death or liver transplantation and the combination thereof was defined as transplant-free survival for survival analysis. 
Non-fatal hepatic events were any episode of hepatic decompensation (defined as ascites, encephalopathy or variceal bleeding) and development of HCC.

Definition of cirrhosis: In case of unequivocal biochemical and/or clinical signs of cirrhosis, no biopsy was performed. These were laboratory evidence of advanced liver disease with hyperbilirubinemia, and/or thrombocytopenia, and/or prolonged prothrombin time together with imaging evidence of cirrhosis and/or ultrasound examination showing irregular liver surface in conjunction with splenomegaly or esophageal varices and/or history of past or recent hepatic decompensation (ascites, variceal bleeding and hepatic encephalopathy). Liver biopsy is generally not performed in our centers in these clinical constellations and subjects fulfilling these criteria at baseline were not included in our cohort. Subjects classified as cirrhosis at baseline were diagnosed on histological grounds. No FibroScan ${ }^{\circ}$ (Echosens, Paris, France) was available at baseline investigations in most subjects (until 2016). At the time of follow-up, cirrhosis was additionally defined clinically as LSM $>20.0 \mathrm{kPa}$. The LSM cut-off was chosen for practicability and to avoid false-positive diagnoses of cirrhosis with high accuracy in the clinical evaluation $[24,25]$.

Cardiovascular endpoints were defined as death from cardiovascular cause (myocardial infraction, stroke and peripheral artery disease), and the history of cerebrovascular stroke, myocardial infarction and acute heart failure and symptomatic peripheral artery disease between the time of liver biopsy and follow-up was counted as non-fatal cardiovascular events. Any diagnosis of an extrahepatic malignancy was counted as fatal or non-fatal malignancy-related event.

\section{Liver Biopsy and Histological Examination}

Liver biopsy specimens with at least $15-18 \mathrm{~mm}$ in length corresponding to a sufficient number of portal fields $(>10)$ were used for histological analysis. All samples evaluated in this study were re-analysed in 2017 for current criteria for diagnosing, grading and staging of NAFLD as described below. Deparaffinized sections $(4 \mu \mathrm{m})$ of each case were processed according to routine protocols and stained with hematoxylin, eosin and Masson's trichrome stain. All slides were evaluated and scored by two pathologists unaware of the clinical data first individually, and in case of discordant results, slides were again analysed conjointly using a multiheaded microscope, and a scoring decision was reached in consensus. Histological grading and staging of NAFLD components were performed as published by Kleiner et al. [26] by application of numerical scores. Accordingly, scores were reported for steatosis $(0-3)$, lobular inflammation (0-3), hepatocellular ballooning ( $0-2)$, Mallory-Denk bodies $(0-2)$, and portal inflammation $(0-1)$. Fibrosis stage was assessed on a 5-step scale including stages 0 (none), 1 (centrilobular or pericellular), 2 (centrilobular plus periportal), 3 (bridging), and 4 (cirrhosis). Ballooned hepatocytes were characterized by a marked increase in size (approximately two times the size of a normal hepatocyte), rounded cell shape and pale staining of the cytoplasm. The diagnostic algorithm for histological classification as NAFLD or NASH was followed as reported by Bedossa et al. [27] in the Steatosis, Activity and Fibrosis (SAF) score.

\section{Statistical Analysis}

Continuous data points are expressed as mean \pm standard deviation. Differences between independent groups were calculated using ANOVA. Categorical data are expressed as numbers (percentage). The chi-square test was applied to calculate univariate differences between groups. Survival curves for 10-year survival were depicted using the KaplanMeier method and differences between groups were calculated using the log-rank test. Univariable and multivariable Cox regression analysis were performed to assess associations with 10-year-mortality. Hazard ratios (HR) and adjusted hazard ratios (aHR) with respective 95\% confidence intervals (CI) were calculated. Four Cox regression models were built, model one was unadjusted, model 2 adjusting for age, gender and fasting glucose at baseline, model three for age, gender, fasting glucose at baseline and fibrosis and model 4 for FIB- 4 score. Fasting glucose was chosen as the surrogate for diabetes at baseline as the diagnosis had not been reliably documented in all subjects. The lean NAFLD group was set as the reference category. All tests were two-sided, and a p-value of $<0.05$ was considered statistically significant. SPSS version 23.0 (IBM, USA) and MedCalc Statistical Software version 19.1.3 (MedCalc Software bv, Ostend, Belgium; https://www.medcalc. org; 2019) were used for all statistical analyses.

\section{RESULTS}

\section{Baseline Characteristics}

A total of 299 subjects ( $28 \%$ female) with a mean age of 49.5 years ( \pm 13.4 ; range: $17-80)$ and a mean BMI of $28.7 \mathrm{~kg} / \mathrm{m} 2$ ( \pm 3.8 ; range: $17.7-41.7$ ) were included. Clinical, biochemical and histological characteristics are summarized in Table I. While age and gender were similar among groups, components of the MetS and ALT increased with BMI. The proportion of subjects with significant fibrosis and the number of subjects with cirrhosis was increased in lean subjects while the proportion of subjects with NASH was not different.

\section{Fatal and Non-fatal Clinical Endpoints}

During the follow-up period 36 deaths occurred in the NAFLD cohorts and 21 in the control group. The leading cause of death in the NAFLD cohorts was liver-related $(n=15)$, followed by extrahepatic malignancy $(\mathrm{n}=7)$ and cardiovascular $(\mathrm{n}=7)$. Seven deaths ( 2 accidents, 2 infectious causes, and 3 chronic lower respiratory diseases) were not classified to one of these groups and not counted in the analysis. Among controls the leading cause of death was extrahepatic malignancy $(n=13)$ followed by cardiovascular $(n=5)$. The comparison between the control group and the NAFLD cohort revealed a significantly worse survival rate and event-free rate in the liver cohort in Kaplan-Meyer analysis (Fig. 1A and 1B; log-rank $\mathrm{p}<0.001$ ). Lean NAFLD patients had a higher mortality rate due to hepatic events and malignant neoplasms, whereas cardiovascular deaths were numerically more frequent in obese patients (Tables II, III and IV), which translated into a significantly worse survival rate among lean NAFLD subjects in KaplanMeyer analysis (Fig. 2A, log-rank $\mathrm{p}<0.05$ ). Overweight patients evidenced lower rates of mortality (HR $=0.34 ; 95 \%$ CI: $0.13-0.89$; $\mathrm{p}=0.03$ ) compared to lean NAFLD (Table IV). 
Table I. Baseline clinical and histological characteristics of the study population

\begin{tabular}{|c|c|c|c|c|c|c|c|}
\hline & $\mathrm{BMI}<25.0$ & BMI 25.0-29.9 & $\mathrm{BMI}>30.0$ & p-value & NAFLD cohort & Control & $\mathrm{p}$ \\
\hline & $\mathrm{n}=39$ & $\mathrm{n}=164$ & $\mathrm{n}=93$ & & $n=299$ & $\mathrm{n}=1013$ & \\
\hline Age (years) & $47.6(14.5)$ & $49.7(13.7)$ & $49.9(12.5)$ & 0.65 & $49.5(13.5)$ & $52.4(5.8)$ & 0.96 \\
\hline Female gender n (\%) & $16(42)$ & $42(26)$ & $26(28)$ & 0.12 & $84(28)$ & $466(64)$ & $<0.001$ \\
\hline Follow up time, months (max) & $84(180)$ & $93(216)$ & $85(204)$ & & $89(216)$ & $109(120)$ & 0.06 \\
\hline Diast. BP (mmHg) & $73.7(9.4)$ & $78.0(11.4)^{*}$ & $84.1(11.4)^{\star} \#$ & 0.002 & $79.5(11.57)$ & $89(12)$ & $<0.001$ \\
\hline Syst. BP (mmHg) & $115.3(13.8)$ & $127.9(17.8)^{*}$ & $134.7(20.8)^{\star} \#$ & 0.002 & $128.7(17.3)$ & $141(19)$ & $<0.001$ \\
\hline BMI $\left(\mathrm{kg} / \mathrm{m}^{2}\right)$ & $23.0(1.5)$ & $27.5(1.5)^{*}$ & $33.2(2.5)^{\star} \#$ & $<0.001$ & $28.7(1.9)$ & $26.9(4)$ & $<0.001$ \\
\hline Triglycerides (mg/dL) & $185.0(176.6)$ & $183.6(125.0)$ & $229.5(258.6)$ & 0.15 & $198.0(125.7)$ & $130(89)$ & $<0.001$ \\
\hline Cholesterol (mg/dL) & $214.4(58.8)$ & $216.0(53.7)$ & $226.7(55.8)$ & 0.290 & $219.1(53.9)$ & $233(42)$ & $<0.001$ \\
\hline HDL-C (mg/dL) & $61.3(29.8)$ & $50.2(15.1)^{*}$ & $47.8(12.8)^{*}$ & $<0.001$ & $50.9(15.4)$ & $60(16)$ & $<0.001$ \\
\hline Fasting glucose (mg/dl) & $90.8(16.5)$ & $105.6(33.4)^{\star}$ & $115.1(34.5)^{* \#}$ & 0.001 & $106.6(33.9)$ & $94(19)$ & $<0.001$ \\
\hline HbAlc (\%) & $5.43(0.43)$ & $6.20(1.24)$ & $6.01(0.43)$ & 0.32 & $6.09(1.04)$ & $5.61(0.5)$ & 0.002 \\
\hline Bilirubin (mg/dL) & $1.0(1.2)$ & $0.9(0.5)$ & $0.8(0.2)$ & 0.168 & $0.9(0.6)$ & & \\
\hline GGT (IU/L) & $164.6(162.1)$ & $133.2(133.1)$ & $190.3(346.1)$ & 0.15 & $155.3(133.5)$ & $22(22)$ & $<0.001$ \\
\hline AST (IU/L) & $47.6(24.8)$ & $43.9(27.6)$ & $51.4(37.8)$ & 0.17 & $46.8(27.9)$ & $12(5)$ & $<0.001$ \\
\hline ALP (IU/L) & $120.0(105.1)$ & $86.4(63.3)^{*}$ & $86.4(79.1)^{*}$ & 0.003 & $90.9(54.9)$ & & \\
\hline ALT (IU/L) & $54.6(35.5)$ & $68.2(51.3)^{*}$ & $85.5(53.5)^{\star \#}$ & 0.003 & $71.8(51.3)$ & $17(10)$ & $<0.001$ \\
\hline Hemoglobin (g/dL) & $14.7(1.7)$ & $15.3(1.5)^{*}$ & $15.6(1.7)^{*}$ & 0.007 & $15.3(1.5)$ & $14.1(1.2)$ & $<0.001$ \\
\hline Platelets (G/L) & $220.1(68.0)$ & $215.2(62.5)$ & $204.6(53.0)$ & 0.291 & $212.5(62.5)$ & $223(51)$ & 0.033 \\
\hline Ferritin (ug/dL) & $567.0(549.1)$ & $473.2(431.2)$ & $596.5(518.1)$ & 0.12 & $523.2(431.1)$ & $188.3(172.9)$ & $<0.001$ \\
\hline FIB-4 & $2.00(2.04)$ & $1.46(1.15)$ & $1.65(1.04)$ & 0.14 & $1.59(1.1 .52)$ & & \\
\hline MetS n (\%) & $11(28)$ & $69(42)$ & $77(81)^{* \#}$ & $<0.001$ & $157(52)$ & $214(21)$ & $<0.001$ \\
\hline Diabetes n (\%) & $1(3)$ & $42(26)^{*}$ & $35(38)^{* \#}$ & $<0.001$ & $78(26)$ & $42(4)$ & $<0.001$ \\
\hline IFG n (\%) & $10(26)$ & $53(32)$ & $40(42)$ & 0.112 & $103(34)$ & $215(21)$ & $<0.001$ \\
\hline Diabetes medication n (\%) & $0(0)$ & $33(20)^{*}$ & $31(33)^{* \#}$ & $<0.001$ & $64(21)$ & $19(2)$ & $<0.001$ \\
\hline Hypertension n (\%) & $14(36)$ & $85(51)$ & $65(70)^{\star \#}$ & 0.001 & $165(55)$ & $656(64)$ & 0.003 \\
\hline Antihypertensive medication $\mathrm{n}(\%)$ & $13(33)$ & $7243)$ & $57(61)^{* \#}$ & 0.004 & $143(48)$ & $166(16)$ & $<0.001$ \\
\hline High TG n (\%) & $15(39)$ & $82(50)$ & $52(56)$ & 0.18 & $150(50)$ & $274(27)$ & $<0.001$ \\
\hline Low HDL-C n (\%) & $13(33)$ & $51(31)$ & $30(32)$ & 0.96 & $94(31)$ & $134(13)$ & $<0.001$ \\
\hline Statins n (\%) & $11(28)$ & $62(38)$ & $42(45)$ & 0.12 & $116(39)$ & $51(5)$ & $<0.001$ \\
\hline Steatosis, $\%$ & $28 \pm 22$ & $27 \pm 19$ & $37 \pm 21$ & 0.001 & $30(21)$ & & \\
\hline Grade $1(5-33 \%)$ & $23(59)$ & $103(63)$ & $35(38)$ & 0.00 & $161(54)$ & & \\
\hline Grade $2(33-66 \%)$ & $13(33)$ & $56(34)$ & $49(53)$ & 0.01 & $118(40)$ & & \\
\hline Grade $3(>66 \%)$ & $3(8)$ & $4(3)$ & $8(9)$ & 0.07 & $15(5)$ & & \\
\hline Hepatocellular ballooning & $11(28)$ & $22(13)$ & $20(22))$ & 0.005 & $53(18)$ & & \\
\hline grade $0 \mathrm{n}(\%)$ & $28(72)$ & $141(87)$ & $72(78)$ & 0.06 & $241(81)$ & & \\
\hline grade1 n (\%) & $4(10)$ & $18(11)$ & $9(10)$ & 0.95 & $31(10)$ & & \\
\hline grade $2 \mathrm{n}(\%)$ & $7(18)$ & $4(2)$ & $11(12)$ & 0.001 & $22(7)$ & & \\
\hline Lobular inflammation $\mathrm{n}(\%)$ & $8(21)$ & $14(8)$ & $14(15)$ & 0.01 & $31(12)$ & & \\
\hline grade $0 \mathrm{n}(\%)$ & $31(79)$ & $149(91)$ & $78(85)$ & 0.08 & $258(86)$ & & \\
\hline grade1 n (\%) & $5(13)$ & $13(8)$ & $13(14)$ & 0.27 & $31(10)$ & & \\
\hline grade $2 \mathrm{n}(\%)$ & $3(8)$ & $1(1)$ & $1(1)$ & 0.007 & $5(2)$ & & \\
\hline Portal inflammation $\mathrm{n}(\%)$ & $6(16)$ & $13(8)$ & $12(13)$ & 0.27 & $31(11)$ & & \\
\hline grade $0 \mathrm{n}(\%)$ & $33(85)$ & $150(92)$ & $80(87)$ & 0.27 & $263(88)$ & & \\
\hline grade $1 \mathrm{n}(\%)$ & $5(13)$ & $13(8)$ & $11(12)$ & 0.46 & $29(10)$ & & \\
\hline grade $2 \mathrm{n}(\%)$ & $1(2)$ & $0(0)$ & $1(1)$ & 0.18 & $2(1)$ & & \\
\hline NASH n (\%) & $9(23)$ & $15(9)$ & $14(15)$ & 0.05 & $38(13)$ & & \\
\hline
\end{tabular}




\begin{tabular}{l} 
Table I (continued) \\
\hline Fibrosis stage \\
Stage 0 n(\%) \\
Stage $1 \mathrm{n}(\%)$
\end{tabular}

Data are means \pm standard deviations or frequencies (\%) p-value assessed by ANOVA with post-hoc analysis or chi-square test; BMI: body mass index; BP: blood pressure; ALT/AST: alanine/aspartate transaminase; ALP: alkaline phosphatase; BMI: body mass index; FIB-4: fibrosis 4 score; GGT: gamma-glutamyl transferase; HDL-C: high density lipoprotein cholesterol; LDL-C: low-density lipoprotein cholesterol; MetS:- metabolic syndrome; IFG: impaired fasting glucose; ${ }^{*}$ significant difference $\mathrm{p}<0.05$ in post-hoc analysis compared to $\mathrm{BMI}<25$; ${ }^{*}$ significant difference $\mathrm{p}<0.05$ in post-hoc analysis compared to BMI 25.0-29.9; NASH: non-alcoholic steatohepatitis.

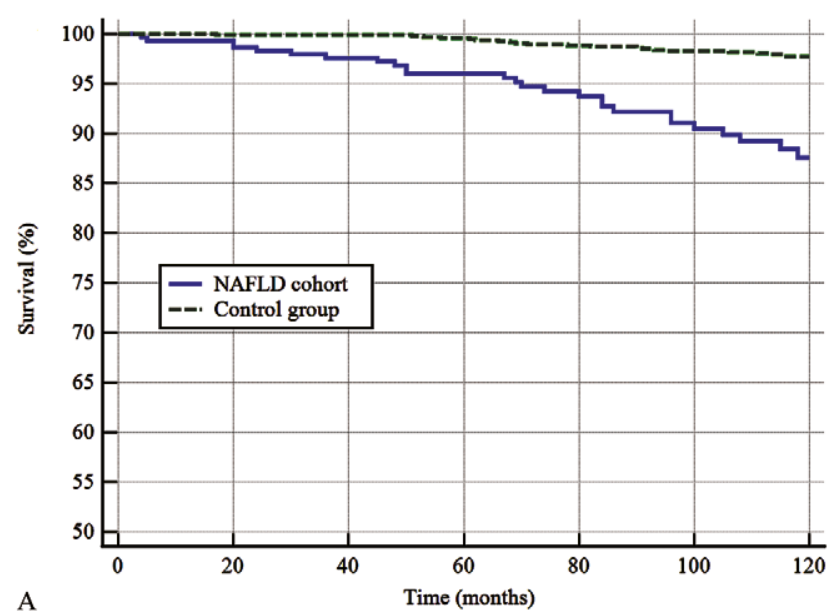

A

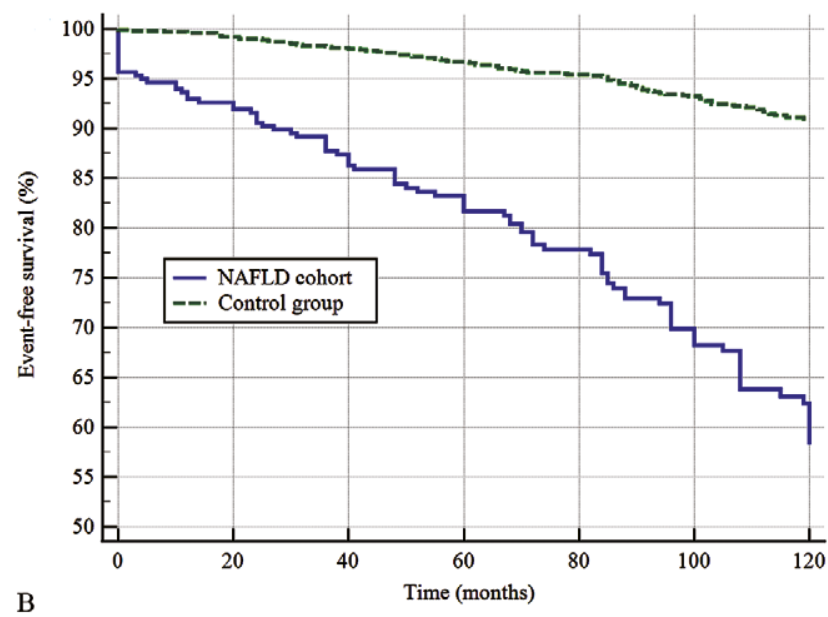

Fig. 1. Cumulative survival (panel A) and cumulative incidence of fatal and non-fatal events combined (B)of the NAFLD cohort and control group.

This association remained significant after adjustment for age, gender, fasting glucose and fibrosis $(\mathrm{aHR}=0.22$; $95 \% \mathrm{CI}$ : 0.07-0.70; $\mathrm{p}=0.01$ ). Compared to obese patients, subjects with lean NAFLD had similar mortality ( $\mathrm{aHR}=0.33$; 95\%CI: 0.081.31; $\mathrm{p}=0.12$ ) after multivariable adjustment (Table IV). The combined numbers of fatal and non-fatal events abolished these differences, thus event-free rates were not different between the NAFLD groups (Fig. 2B). A cox regression model showed significantly higher mortality with higher FIB-4 scores among all NAFLD cohorts $(\mathrm{aHR}=1.47$; 95\%CI: 1.329 -
Table II. Absolute number of fatal hepatic, cardiovascular and malignant events in each BMI category and the control group. P-value denotes significance as calculate by chi-square.

\begin{tabular}{|c|c|c|c|c|c|}
\hline Fatal events & $\begin{array}{c}\mathrm{BMI}<25 \\
(\mathrm{n}=39)\end{array}$ & $\begin{array}{c}\text { BMI 25-30 } \\
(n=164)\end{array}$ & $\begin{array}{c}\mathrm{BMI}>30 \\
(\mathrm{n}=93)\end{array}$ & $\begin{array}{c}\text { Control } \\
(\mathrm{n}=1013)\end{array}$ & $\mathrm{p}$ \\
\hline Hepatic n (\%) & $4(11)$ & $7(4)$ & $4(4)$ & $0(0)$ & $<0.001$ \\
\hline $\begin{array}{l}\text { Cardiovascular } \\
\mathrm{n}(\%)\end{array}$ & $1(3)$ & $1(1)$ & $5(5)$ & $5(0.5)$ & $<0.001$ \\
\hline $\begin{array}{l}\text { Extrahepatic } \\
\text { malignancy } \\
\mathrm{n}(\%)\end{array}$ & $2(5)$ & $4(2)$ & $1(1)$ & $13(1)$ & 0.19 \\
\hline Other n (\%) & $2(5)$ & $3(2)$ & $2(2)$ & $3(0.3)$ & 0.001 \\
\hline Total & $9(24)$ & $15(9)$ & $12(13)$ & $21(2)$ & $<0.001$ \\
\hline
\end{tabular}

Table III. Absolute number of fatal and non-fatal hepatic, cardiovascular and malignant events combined in each BMI stratum and control group. $\mathrm{P}$-value denotes significance as calculate by chi-square.

\begin{tabular}{|c|c|c|c|c|c|}
\hline $\begin{array}{l}\text { Fatal and non- } \\
\text { fatal events }\end{array}$ & $\begin{array}{l}\mathrm{BMI}<25 \\
(\mathrm{n}=39)\end{array}$ & $\begin{array}{l}\text { BMI } \\
25-30 \\
(n=164)\end{array}$ & $\begin{array}{l}\mathrm{BMI}>30 \\
(\mathrm{n}=93)\end{array}$ & $\begin{array}{l}\text { Control } \\
(\mathrm{n}=1013)\end{array}$ & $\mathrm{p}$ \\
\hline Hepatic n (\%) & $5(13)$ & $21(13)$ & $7(7)$ & $0(0)$ & $<0.001$ \\
\hline $\begin{array}{l}\text { Cardiovascular } \\
\mathrm{n}(\%)\end{array}$ & $6(15)$ & $24(15)$ & $25(26)$ & $41(4)$ & $<0.001$ \\
\hline $\begin{array}{l}\text { Extrahepatic } \\
\text { malignancy } \\
\mathrm{n}(\%)\end{array}$ & $3(8)$ & $9(6)$ & $6(6)$ & $41(4)$ & 0.46 \\
\hline Other n (\%) & $2(5)$ & $3(2)$ & $3(3)$ & $3(0.3)$ & $<0.001$ \\
\hline Total & $16(41)$ & $57(36)$ & $41(42)$ & $84(8)$ & $<0.001$ \\
\hline
\end{tabular}

1.624; $\mathrm{p}<0.001)$. Furthermore, we did not detect a significant effect of metformin, other antidiabetic drugs, statins or antihypertensive medication at baseline on fatal and non-fatal outcomes in this cohort.

\section{DISCUSSION}

In this study, we report analyze the natural outcome of a regional NAFLD cohort with particular focus on the subgroup of lean NAFLD. We confirm (1) that NAFLD subjects had a higher risk of fatal and non-fatal events compared to the background population and (2) that lean NAFLD subjects had a higher likelihood of dying from liver-related causes compared to overweight and obese NAFLD subjects. These data support that lean subjects deserve particular clinical attention with regard to follow-up when receiving the diagnosis of NAFLD. 
Table IV. Hazard ratios for overall mortality stratified per BMI category with BMI $<25.0$ as reference category.

\begin{tabular}{lccccccccc}
\hline $\begin{array}{l}\text { Hazard for } \\
\text { mortality }\end{array}$ & Model 1 & \multicolumn{7}{c}{ Model 2 } \\
\hline & HR & $95 \%$ CI & p-value & aHR & $95 \%$ CI & p-value & aHR & $95 \%$ CI & p-value \\
BMI $<25$ & (ref.) & & & (ref.) & & & (ref.) & & \\
BMI $25-30$ & 0.34 & $0.13-0.89$ & 0.03 & 0.21 & $0.07-0.62$ & 0.005 & 0.22 & $0.07-0.70$ & 0.01 \\
BMI $\geq 30$ & 0.38 & $0.13-1.07$ & 0.07 & 0.22 & $0.06-0.76$ & 0.02 & 0.33 & $0.08-1.31$ & 0.12 \\
\hline
\end{tabular}

Model 1 shows unadjusted estimates, Model 2 shows adjustment for age sex, fasting glucose Model 3 shows adjustment for age, sex, fasting glucose and fibrosis $0-4$
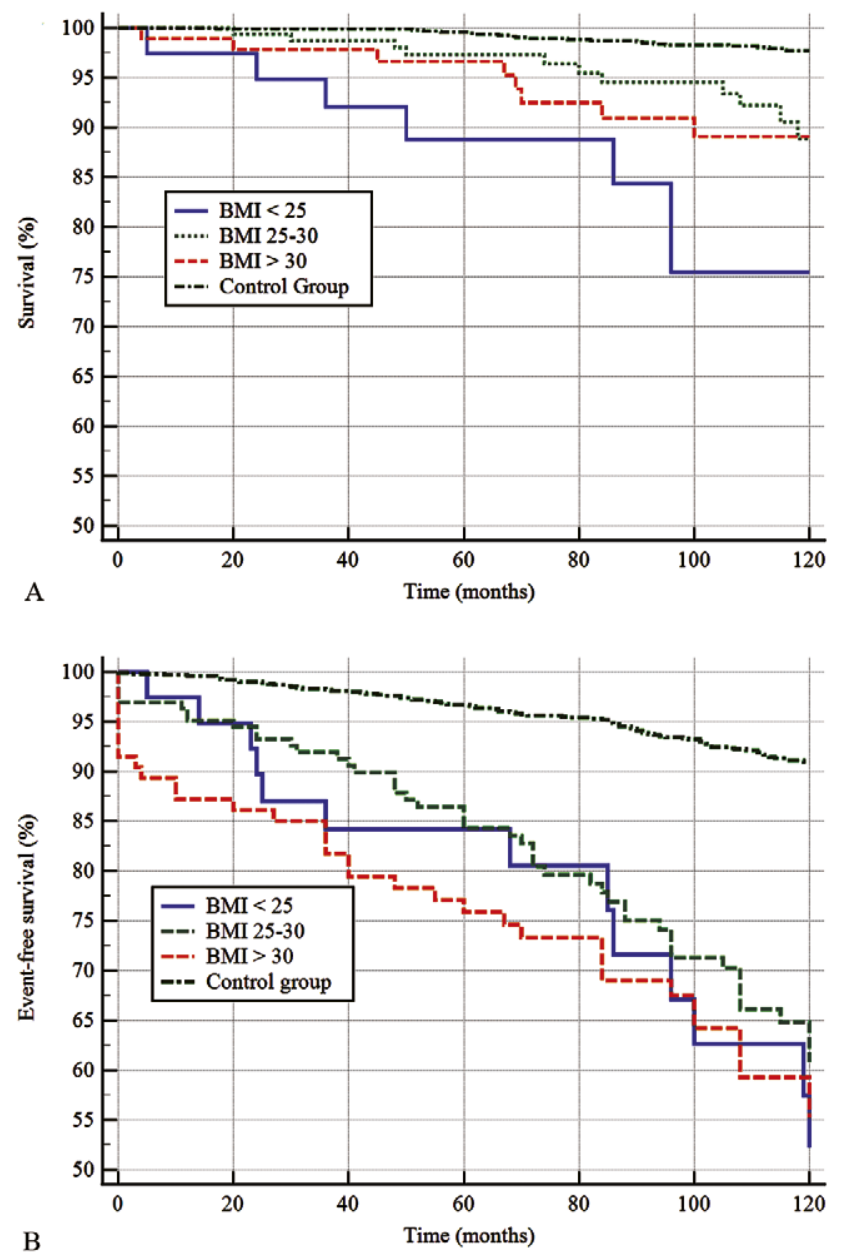

Fig. 2. Cumulative survival (panel A) and cumulative incidence of fatal and non-fatal events combined (B)of all groups.

The syndrome of lean NAFLD has received increasing attention recently due to the observed pathophysiological differences compared to the typical obesity-related NAFLD phenotype [5]. On the one hand, lean NAFLD subjects present with a lower cardiometabolic risk profile compared to overweight or obese subjects and histological severity and natural outcome seem to worsen with higher degrees of obesity. As most of these studies have been performed in Asians, the transferability of these observations to Caucasian populations is uncertain as discussed by Sookian et al. [10]. On the other hand, studies with a designated focus on the lean NAFLD subgroup, have suggested that the histological phenotype, as well as the clinical outcome, may be unexpectedly severe $[13,28$, 29]. Our data are in line with the study by Hagström et al. [13] who reported a specifically increased risk of liver-associated death in the lean subgroup in a Swedish cohort. Particularly informative genetic data suggest that in the lean subgroup an adverse combination of adipose tissue dysfunction and hepatic vulnerability may coincide culminating in liver disease even in the absence of excess total body weight already at a lower degree of clinically manifest metabolic derangements. From a genetic point of view, this is supported by genes implicated in the rare lipodystrophy syndromes [30] as well as liver-specific risk variants associated with intrahepatic lipid homeostasis like PNPLA3 or TM6SF2 to have a role in lean NAFLD $[5,31]$.

It is a limitation of our study that systematic data on lifestyle and diet were not available owing to the retrospective clinical design. Some detrimental dietary habits may have a role in lean NAFLD as it has been proposed for fructose consumption or dietary fat composition with a preponderance of non-vegetable and trans-fats [32]. Furthermore, we are unable to exclude a role for alcohol consumption in the progression of liver disease among lean subjects which is a delicate matter in the work-up of a fatty liver disease patient as underreporting is common [33]. We have aimed to eliminate the potential bias of different alcohol consumption between groups as at baseline and follow-up all patients have been seen by clinically experienced hepatologists where a standard procedure on asking alcohol consumption is followed, together with assessment of laboratory parameters indicative of alcohol consumption. However, the role of low and moderate alcohol consumption as a factor for initiation or progression of NAFLD has not been solved conclusively so far [34]. Some researchers suggested that even lower amounts of alcohol consumption might contribute to metabolic diseases within limits generally accepted as not detrimental to health [35]. While other NAFLD prevalence studies have reported a negative correlation between modest alcohol consumption and NAFLD [36]. Further, the relationship between alcohol and mortality in NAFLD subjects is multifactorial and complex, since modest alcohol consumption is also negatively associated with e.g. cardiovascular risk and those risk factors cannot be adequately adjusted for in a retrospective analysis. Alas, the role of alcohol consumption on cardiovascular mortality has not been investigated specifically in NAFLD cohorts. Additionally, associations derived from prevalence studies have limited application to patients with established NAFLD who present to clinical work-up. In this setting, the presence of significant fibrosis $(\geq \mathrm{F} 2)$ is the key finding because these subjects are estimated to have progressive disease. Thus, particularly prospective cohort studies of disease progression are important from the patient's standpoint, which may elucidate the role of alcohol consumption on the further course of the disease in 
established NAFLD including cardiovascular, malignancyrelated or hepatic endpoints.

It is a key limitation of our study that the presence of visceral obesity (waist circumference) was not available at baseline as this is most closely related to insulin resistance which is probably the key factor for onset and progression of NAFLD [37]. A further limitation is the fact that genetic data (PNPLA3, TM6SF2) as an established co-factor for disease progression was not available and can therefore not be accounted for.

\section{CONCLUSIONS}

Our data add evidence to the observations that lean NAFLD patients have a high risk of liver-related endpoints, indicating that these subjects may benefit from adapted clinical follow-up regarding progression of liver disease to cirrhosis, development of HCC or screening for gastroesophageal varices. However, larger studies are required to confirm our observations before recommendations on clinical management can be derived.

Our findings support the notion that lean NAFLD represents a disease entity different from classical obesity related NAFLD with regard to mechanisms of disease mechanisms and also the natural course of the disease.

\section{Conflicts of interest: None to declare.}

Author contributions: E.A: conceived and designed the study. A.F. analyzed data, drafted and wrote the manuscript. B.W, G.S., S.Z., D.N., S.K.E, M.S., B.P recruited patients and collected data. H.H. K.S. performed the histopathological analysis. C.D. recruited patients and revised the manuscript for important intellectual content: E.A analyzed data analysis outlined and revised the manuscript.

Acknowledgments: Elmar Aigner is supported by PMU Research Fund PMU-FFF (E-18/27/141-AIS and E-15/21/108-AIE). Financial support from SPAR Austria to Christian Datz is gratefully acknowledged.

\section{REFERENCES}

1. Loomba R, Sanyal AJ. The global NAFLD epidemic. Nat Rev Gastroenterol Hepatol 2013;10:686-690. doi:10.1038/nrgastro.2013.171

2. Kim CH, Younossi ZM. Nonalcoholic fatty liver disease: a manifestation of the metabolic syndrome. Cleve Clin J Med 2008;75:721-728.

.3 Das K, Chowdhury A. Lean NASH: distinctiveness and clinical implication. Hepatol Int 2013;7 Suppl 2:806-813. doi:10.1007/s12072013-9477-5

4. Fan JG, Kim SU, Wong VWS. New trends on obesity and NAFLD in Asia. J Hepatol 2017;67:862-873. doi:10.1016/j.jhep.2017.06.003

5. Feldman A, Eder SK, Felder TK, et al. Clinical and metabolic characterization of lean Caucasian subjects with non-alcoholic fatty liver. Am J Gastroenterol 2017;112:102-110. doi:10.1038/ ajg.2016.318

.6 Chen VL, Wright AP, Halligan B, et al. Body composition and genetic lipodystrophy risk score associate with nonalcoholic fatty liver disease and liver fibrosis. Hepatol Commun 2019;3:1073-1084. doi:10.1002/ hep4.1391
7. Lotta LA, Gulati P, Day FR, et al. Integrative genomic analysis implicates limited peripheral adipose storage capacity in the pathogenesis of human insulin resistance. Nat Genet 2017;49:17-26. doi:10.1038/ ng. 3714

8. Machado M, Marques-Vidal P, Cortez-Pinto H. Hepatic histology in obese patients undergoing bariatric surgery. J Hepatol 2006;45:600-606. doi:10.1016/j.jhep.2006.06.013

9. Festi D, Colecchia A, Sacco T, Bondi M, Roda E, Marchesini G. Hepatic steatosis in obese patients: clinical aspects and prognostic significance. Obes Rev 2004;5:27-42. doi:10.1111/j.1467-789x.2004.00126.x

10. Sookoian S, Pirola CJ. Systematic review with meta-analysis: risk factors for non-alcoholic fatty liver disease suggest a shared altered metabolic and cardiovascular profile between lean and obese patients. Aliment Pharmacol Ther 2017;46:85-95. doi:10.1111/apt.14112

11. Vernon G, Baranova A, Younossi Z. Systematic review: the epidemiology and natural history of non-alcoholic fatty liver disease and non-alcoholic steatohepatitis in adults. Aliment Pharmacol Ther 2011;34:274-285. doi:10.1111/j.1365-2036.2011.04724.x

12. Denkmayr L, Feldman A, Stechemesser L, et al. Lean patients with nonalcoholic fatty liver disease have a severe histological phenotype similar to obese patients. J Clin Med 2018;7:562. doi:10.3390/jcm7120562

13. Hagstrom H, Nasr P, Ekstedt M, et al. Risk for development of severe liver disease in lean patients with nonalcoholic fatty liver disease: A longterm follow-up study. Hepatol Commun 2018;2:48-57. doi:10.1002/ hep 4.1124

14. Aung K, Lorenzo C, Hinojosa MA, Haffner SM. Risk of developing diabetes and cardiovascular disease in metabolically unhealthy normalweight and metabolically healthy obese individuals. J Clin Endocrinol Metab 2014;99:462-468. doi:10.1210/jc.2013-2832

15. Cataldi M, Citro V, Resnati C, Manco F, Tarantino G. New Avenues for Treatment and Prevention of Drug-Induced Steatosis and Steatohepatitis: Much More Than Antioxidants. Adv Ther 2021;38:20942113. doi:10.1007/s12325-021-01669-y

16. Roeb E, Steffen $\mathrm{HM}$, Bantel $\mathrm{H}$, et al. S2k Guideline non-alcoholic fatty liver disease. Z Gastroenterol 2015;53:668-723. doi:10.1055/s-0035-1553193

17. Eder SK, Feldman A, Strebinger G, et al. Mesenchymal iron deposition is associated with adverse long-term outcome in non-alcoholic fatty liver disease. Liver Int 2020;40:1872-1882. doi:10.1111/liv.14503

18. Heid IM, Wagner SA, Gohlke H, et al. Genetic architecture of the APM1 gene and its influence on adiponectin plasma levels and parameters of the metabolic syndrome in 1,727 healthy Caucasians. Diabetes 2006;55:375-384. doi:10.2337/diabetes.55.02.06.db05-0747

19. Kedenko L, Lamina C, Kedenko I, et al. Genetic polymorphisms at SIRT1 and FOXO1 are associated with carotid atherosclerosis in the SAPHIR cohort. BMC Med Genet 2014;15:112. doi:10.1186/s12881014-0112-7

20. Grundy SM, Cleeman JI, Merz CNB, et al. Implications of recent clinical trials for the national cholesterol education program adult treatment panel III guidelines. J Am Coll Cardiol 2004;44:720-732. doi:10.1016/j. jacc.2004.07.001

21. Ärnlöv J, Sundström J, Ingelsson E, Lind L. Impact of BMI and the metabolic syndrome on the risk of diabetes in middle-aged men. Diabetes Care 2011;34:61-65. doi:10.2337/dc10-0955

22. Sattar N, Gaw A, Scherbakova O, et al. Metabolic syndrome with and without $\mathrm{C}$-reactive protein as a predictor of coronary heart disease and diabetes in the West of Scotland Coronary Prevention Study. Circulation 2003;108:414-419. doi:10.1161/01.CIR.0000080897.52664.94

23. Sterling RK, Lissen E, Clumeck N, et al. Development of a simple noninvasive index to predict significant fibrosis in patients with 
HIV/HCV coinfection. Hepatology 2006;43:1317-1325. doi:10.1002/ hep. 21178

24. De Franchis R; Baveno VI Faculty. Expanding consensus in portal hypertension: Report of the Baveno VI Consensus Workshop: Stratifying risk and individualizing care for portal hypertension. J Hepatol 2015;63:743-752. doi:10.1016/j.jhep.2015.05.022

25. Siddiqui MS, Vuppalanchi R, Van Natta ML, et al. Vibration-controlled transient elastography to assess fibrosis and steatosis in patients with nonalcoholic fatty liver disease. Clin Gastroenterol Hepatol 2019;17:156-163.e2. doi:10.1016/j.cgh.2018.04.043

26. Kleiner DE, Brunt EM, Van Natta M, et al. Design and validation of a histological scoring system for nonalcoholic fatty liver disease. Hepatology 2005;41:1313-1321. doi:10.1002/hep.20701

27. Bedossa P, Poitou C, Veyrie N, et al. Histopathological algorithm and scoring system for evaluation of liver lesions in morbidly obese patients. Hepatology 2012;56:1751-1759. doi:10.1002/hep.25889

28. Zou B, Yeo YH, Nguyen VH, Cheung R, Ingelsson E, Nguyen MH. Prevalence, characteristics and mortality outcomes of obese, nonobese and lean NAFLD in the United States, 1999-2016. J Intern Med 2020;288:139-151. doi:10.1111/joim.13069

29. Cruz ACD, Bugianesi E, George J, et al. 379 characteristics and longterm prognosis of lean patients with nonalcoholic fatty liver disease. Gastroenterology 2014;146:S-909. doi:10.1016/S0016-5085(14)63307-2

30. Bagias C, Xiarchou A, Bargiota A, Tigas S. Familial Partial Lipodystrophy (FPLD): Recent Insights. Diabetes Metab Syndr Obes 2020;13:1531-1544. doi:10.2147/DMSO.S206053
31. Fracanzani AL, Petta S, Lombardi R, et al. Liver and cardiovascular damage in patients with lean nonalcoholic fatty liver disease, and association with visceral obesity. Clin Gastroenterol Hepatol 2017;15:1604-1611.e1. doi:10.1016/j.cgh.2017.04.045

32. Assy N, Nasser G, Kamayse I, et al. Soft drink consumption linked with fatty liver in the absence of traditional risk factors. Can J Gastroenterol Hepatol 2008;22:811-816. doi:10.1155/2008/810961

33. Stockwell T, Donath S, Cooper-Stanbury M, Chikritzhs T, Catalano P, Mateo C. Under-reporting of alcohol consumption in household surveys: a comparison of quantity-frequency, graduated-frequency and recent recall. Addiction 2004;99:1024-1033. doi:10.1111/j.13600443.2004.00815. $\mathrm{x}$

34. Sookoian S, Pirola CJ. How safe is moderate alcohol consumption in overweight and obese individuals? Gastroenterology 2016;150:16981703.e2. doi:10.1053/j.gastro.2016.01.002

35. VanWagner LB, Ning H, Allen NB, et al. Alcohol use and cardiovascular disease risk in patients with nonalcoholic fatty liver disease. Gastroenterology 2017;153:1260-1272.e3. doi:10.1053/j. gastro.2017.08.012

36. Sookoian S, Castaño GO, Pirola CJ. Modest alcohol consumption decreases the risk of non-alcoholic fatty liver disease: a metaanalysis of 43175 individuals. Gut 2014;63:530-532. doi:10.1136/ gutjnl-2013-305718

37. Tarantino G, Citro V, Capone D. Nonalcoholic fatty liver disease a challenge from mechanisms to therapy. J Clin Med 2020;9:15. doi:10.3390/jcm9010015 